- The principles of colour perception, explained in terms of the interactions between a light source, an object and an observer.

- Fundamental aspects of light sources.

- The ways in which an object modifies the light reflected from it.

- Characteristics of the observer and principles of human colour vision

- The essentials of colorimetry and instrumental colour measurement.

\title{
Colour in relation to dentistry. Fundamentals of colour science
}

\author{
S. M. Burkinshaw ${ }^{1}$
}

The past decade or so has witnessed a marked increase in consumer awareness in virtually every aspect of modern life. The concomitant rise in consumer expectations has extended, of course, to dentistry and, in particular, to the more aesthetic aspects of the discipline, namely shade matching and tooth whiteness. As a consequence, various instrumental methods of shade matching have been launched commercially in recent years; in addition, considerable, commercial research effort is currently being focused on instrumental methods of assessing tooth 'whitening'. Such interest in 'aesthetic dentistry' has, in turn, resulted in the author's laboratories having received myriad enquiries from dental researchers in relation to colour perception, colour theory and instrumental colour measurement. Despite the importance of colour in relation to dentistry and the current, very high level of research interest in the subject, remarkably few, if any, references have appeared that concern the application of colour theory to dentistry; furthermore, the colorimetric aspects of shade matching and tooth whitening have received virtually no discussion in relevant journals. The high level of interest in colour in dentistry coupled with the paucity of publications in this particular area, prompted the research work presented here.

This paper concerns both dental shade matching and tooth whitening. The first part of the paper deals with the basics of colour perception, colour theory and instrumental colour measurement, in terms of three key elements, namely light source, object and observer. The fundamental aspects of light sources, how they interact with objects and the manner in which these interactions are interpreted through human colour vision are discussed. The principles of colorimetry are then considered and the functioning of instrumental colour measuring instruments are discussed.

Subsequent papers will review the various approaches that have been used for shade matching, including methods that have been devised for visual shade matching and the various systems that use instrumental methods of shade matching. Research find-

${ }^{1}$ Head of the School of Design, Dean of Faculty, The University, Leeds LS2 9JT, UK Email:s.m.burkinshaw@leeds.ac.uk

\section{Refereed Paper}

Received 13.03.03; Accepted 11.04.03

doi:10.1038/sj.bdj.4810880

() British Dental Journal 2004; 196: 33-41 ings will be presented that identify the variables (eg lighting, shade guides) which can influence the accuracy of visual shade matching. The concept of instrumental shade matching will be explained in relation to the principles of colour perception, colour theory and instrumental colour measurement. Finally, the subject of tooth whitening will be discussed, from a colorimetric viewpoint.

\section{INTRODUCTION}

Of our five senses, vision is probably that which provides the normally sighted with the greatest amount of information about their (known) surroundings and colour plays a major role in this information gathering. Colour is of course all around us, in the natural world from multicoloured rainbows to the jet blackness of space, bright yellow peppers to luscious ripe, red strawberries and vibrantly coloured macaws to snow white polar bears. Man has sought to use colour in every facet of life, in cultural aspects such as aesthetics (clothing and furnishings), cosmetics, design and art through to 'necessities' such as identification (fluorescent clothing), warning (yellow and black stripes) and control (traffic lights). Colour is used to entice us to eat, drink, work, play and to buy those consumer products that are just so essential for modern life.

The concept of colour has fascinated man since time immemorial. It is well known that people expressed themselves via cave paintings some 15,000-30,000 years ago. Intricately coloured potteries dating from 5,000 years ago are available and, from around 1,500BC, advances began to be made in the manufacture and application of colorants in Egypt and Mesopotamia. Later, as Hellenism culture developed, the first theories of colour and aesthetics flourished in 250-500BC; these persisted until the seventeenth century and the quest of European scholars for scientific understanding. Our current ideas of the theoretical basis of colour mixing and of colour vision have as their basis, fundamental experiments and philosophies developed during the nineteenth and twentieth centuries.

Colour is a highly faceted phenomenon which spans nature, science and culture, as evidenced by the fact that the Concise Oxford Dictionary lists no fewer than two different meanings for the verb 'colour' and thirteen for the noun. ${ }^{1}$ This paper deals only with the science of colour; a variety of texts are available concerning colour in art, culture and life. ${ }^{2-7}$ 


\section{FUNDAMENTALS OF COLOUR SCIENCE}

Nassau has distinguished fifteen specific, colour-producing mechanisms which can be collected into five groups, in which the colour originates from: ${ }^{7}$

- Simple excitations and vibrations (flames, vapour lamps, water)

- Transitions involving ligand (crystal) fields (transition metal compounds, fluorescence, emerald)

- Transitions between molecular orbitals (dyes, sapphire)

- Transitions involving energy bands (metals, semiconductors, phosphors), and

- Geometric and optical effects (rainbows, blue sky, soap bubbles).

Such is the extensive nature of this particular aspect of colour that the discussion of these topics cannot be covered in an article of this length and the reader is directed to the original work. ${ }^{7}$

The science of colour and coloured materials involves chemistry, biology and physics. However, the science of colour differs from other areas of science insofar as while quantities such as mass or volume are intrinsic properties of an object that are identical for all observers, the description of the colour of an object refers to a sensation experienced by a particular observer. In simple terms, the perception of the colour of a reflective object depends upon the combination of three elements (Fig. 1), namely a light source, an object and an observer which are related:

- Light source: illuminates the object

- Object: reflects, absorbs or transmits the incident light to the observer

- Observer: perceives the reflected light.

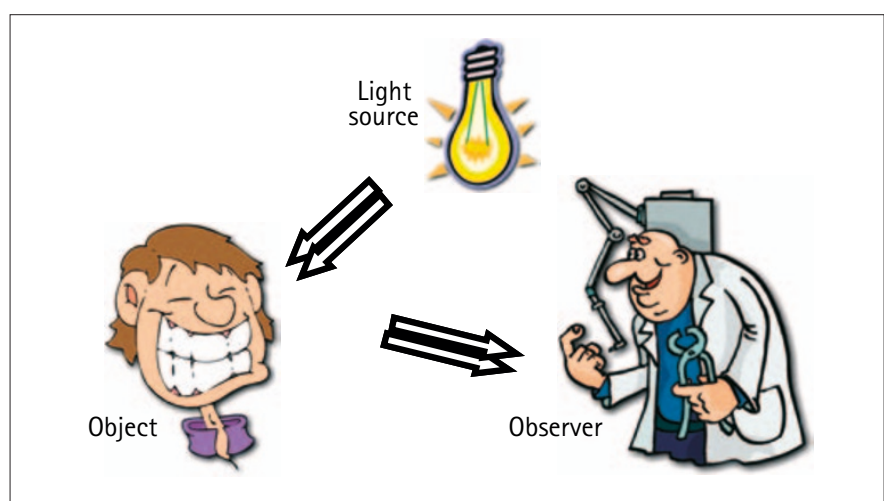

Fig. 1 The three components of colour

The properties of the object, especially how it modifies the light reflected from it, contribute to the perceived colour; an object viewed under a different light source or, when viewed by a different observer, may appear different in colour. Obviously, it follows that if we take any one of these three components away, there is no colour.

It is commonly held that such is the calibre of the human eye/brain system that the normally sighted can distinguish some 3-5 million different colours. However, although we can visually distinguish different colours, the verbal description of different colours is an entirely different situation; the mere thought of having to distinguish verbally between say two million red shades or one million blue shades is disconcerting to say the least. Colour has three dimensions and, as such, comprises a three-dimensional system just like length, width and depth. The three attributes of colour are hue, lightness and chroma (Fig. 2).

Hue is the term we use to distinguish one group of similar colours from another and is the name of a colour (or colour family) such as red, yellow, orange and blue. Chroma is the quality we use to distinguish saturated colours from weak, pastel ones and lightness is the term we use to distinguish lighter shades from darker

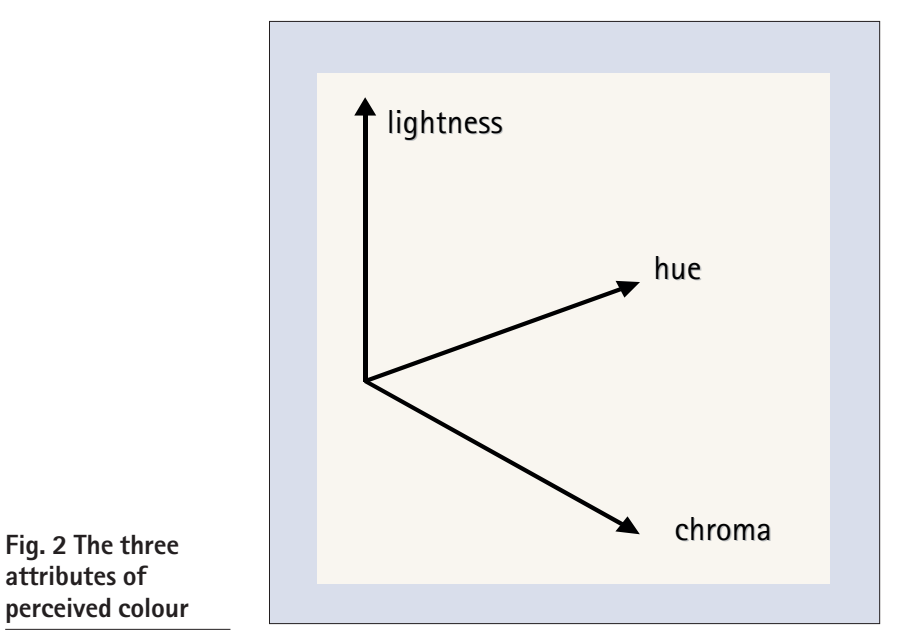

ones. A typical vocabulary for describing the three dimensions of colour is:

- Hue: red, green, blue, etc

- Lightness: dark, light

- Chroma: intense, dull.

This enables us to describe colours more accurately. For instance, instead of describing the blue portion of the US flag as, simply, 'blue', it is more accurately described as a dark, dull blue colour. The three-dimensional nature of colour will be discussed later.

\section{LIGHT SOURCES}

It is generally considered that the foundations of modern colour science were laid by the English mathematician and philosopher, Isaac Newton (1642-1747). In 1665, Newton began work on three of his colossal scientific accomplishments, namely the theory of gravitation (theoretical physics), development of calculus (mathematics) and the colour composition of light (experimental physics). Glass prisms were relatively common in the early part of the seventeenth century, and, it was known (as nowadays by most schoolchildren) before Newton began his work on experimental physics, that visible light (sunlight) could be split into the colours of the rainbow by a prism. Newton considered that only seven colours were present in his visible 'spectrum' (red, orange, yellow, green, blue, indigo and violet) whereas there are in fact many more colours in the visible spectrum. He also showed that the colour of one region of the spectrum could not be dispersed further by passage through a second prism. Importantly, by using two prisms, Newton demonstrated that a beam of sunlight which had been dispersed into a spectrum by one prism, could be merged back into white light by passage through a second prism. In doing this, Newton proved that achromatic light (colourless or white light) is a combination of all spectral colours.

\section{Light}

Light is a certain kind of electromagnetic radiation, a term for the (as yet not fully understood) phenomenon of energy transfer through space. Electromagnetic radiation travels in the form of both:

- Waves: light travels at a constant speed, c, in a vacuum, of $2.99792458 \times 10^{8} \mathrm{~m} \mathrm{~s}^{-1}$ and its energy content is expressed in terms of wavelength $\lambda$ and frequency $v$;

- Quanta: particle-like packets of energy (aka photons) whose energy content is expressed in terms of $\mathrm{eV}\left(1 \mathrm{eV} \sim 8,066 \mathrm{~cm}^{-1}\right.$ $\sim 100 \mathrm{~kJ} \mathrm{~mol}^{-1}$ ).

Electromagnetic radiation varies markedly from radio frequency $\left(\lambda=3 \mathrm{~km} ; v<10^{9} \mathrm{~Hz}\right)$ through infra red $\left(\lambda=0.03 \mathrm{~mm} ; v=10^{13} \mathrm{~Hz}\right)$ and $\mathrm{x}$-rays $\left(\lambda=3 \mathrm{~nm} ; v=10^{17} \mathrm{~Hz}\right)$ to gamma rays $(\lambda=3 \mathrm{pm}$; 
$\left.v>10^{20} \mathrm{~Hz}\right)$ and so deviates in energy content $\left(\mathrm{mm}=10^{-3} \mathrm{~m}\right.$; $\mathrm{nm}=10^{-9} \mathrm{~m} ; \mathrm{pm}=10^{-12} \mathrm{~m}$ ). The region of the electromagnetic spectrum to which the human eye is sensitive extends from around $380 \mathrm{~nm}$ to $780 \mathrm{~nm}\left(\sim 8 \times 10^{14} \mathrm{~Hz}\right.$ to $\left.4 \times 10^{14} \mathrm{~Hz}\right)$, corresponding to an energy content of approximately $3.1 \mathrm{eV}$ (violet) to $1.8 \mathrm{eV}$ (red).

Electromagnetic radiation that is referred to as 'light' includes natural light sources, such as the sun and flames, as well as artificial light sources such as incandescent lamps and fluorescent lights. As the amount of radiation emitted by such light sources varies from wavelength to wavelength for the different sources, the different sources vary in the colour of the light they emit, from the bluish white of daylight to the yellowish white of tungsten light.

'Light' is usually produced by incandescence (the emission of light from a hot body). The most important incandescent body is the sun. However, the light from the sun that we see on earth has undergone scattering and absorption in our atmosphere; the nature of daylight depends on several factors such as the latitude of the observation, the season of the year, the weather conditions and the time of day. Furthermore, daylight has two components, namely sunlight and skylight which can differ considerably in terms of the quantity of light at different wavelengths. Indeed, the spectral energy distribution (SED) of daylight, which is a form of polychromatic light (electromagnetic radiation of many wavelengths) varies from the bright blue of north skylight to the distinct yellow of direct sunlight (Fig. 3). This impacts on the viewing of coloured objects and colour matching generally. For instance, north skylight imparts a crisp blueness to an object whereas the same object, when viewed under direct sunlight, will take on a soft, yellow cast.

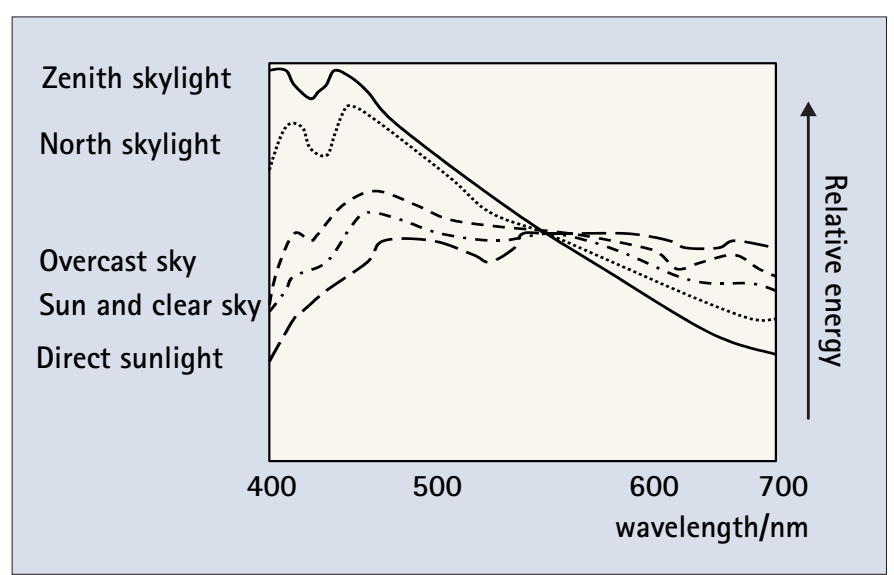

Fig. 3 SED of different forms of daylight

\section{Illuminants}

Owing to the inherent variability of natural daylight, if we are to accurately and reproducibly judge colour, it is necessary to define precisely the light source and its spectral distribution. This was done in 1931 by the Commission Internationale de l'Eclairage (CIE) which proposed the use of standard illuminants. To clarify the situation, a light source (such as a candle or the sun) is a physical emitter of light whereas an illuminant is a set of relative energies over a range of wavelengths that describes given illumination conditions. In simpler terms, a light source can be turned on or off and used to view an object while an illuminant is a numerical description of a light source.

To quantify the energy distribution of an illuminant, black body radiation is used. All objects are continually absorbing and emitting radiation and a black body is a theoretical perfect absorber and emitter of energy (the best approximation to a theoretical black body is a cavity, with insulated walls and a very small ori- fice). Although in practice, no substances approach very closely this ideal over an extended range of wavelengths, black body temperature (expressed in $\mathrm{K}$ ) is used commonly to provide a qualitative description of the colour quality of the emission of a light source even though the emission spectrum may be unlike that of a black body. Some incandescent sources (eg a tungsten filament lamps) have a SED close to (but not identical to) that of a black body radiator; this offers the potential for expressing the colour of the lamp's radiation using the temperature of a black body radiator. In terms of the standard illuminants devised in 1931 by the CIE, Standard Illuminant A, which is typical of light emitted from a tungsten filament lamp, is now defined as the SED of a black body at 2,856 K. The temperature of the black body is in this case known as its colour temperature.

However, for many light sources (eg fluorescent lamps), the SED does not closely enough resemble that of a black body radiator in which case the term correlated colour temperature is used, this being the temperature of a black body whose colour is closest to that of the source. Another standard illuminant devised by the CIE in 1931, Standard Illuminant B, which was intended to simulate direct noon sunlight, is defined as having a correlated colour temperature of $4,874 \mathrm{~K}$ while Standard Illuminant $C$, which was intended to represent average daylight, has a correlated colour temperature of 6,774 K. While each of the above illuminants can be simulated physically (eg by using heated tungsten filament with and without filters) the CIE devised further illuminants (the ' $\mathrm{D}$ ' series of daylight simulating illuminants) that represent different phases of daylight, for which, at present, no physical sources have been recommended. As mentioned earlier, the SED and thus the colour temperature, of daylight varies. For example, direct sunlight has a yellow cast and low colour temperature whereas an overcast sky has a colour temperature of around $6,000 \mathrm{~K}$. Colour temperatures range from around 5,500 $\mathrm{K}$ in the case of a clear day (sun + skylight), 7,500 K for indoor north skylight and 6,500 K for average daylight (diffuse skylight without direct sunlight); indeed colour temperatures for daylight can vary from as low as $2,000 \mathrm{~K}$ in the morning to $10,000 \mathrm{~K}$ and above in late afternoon. To address the variability of daylight, the CIE has defined an 'average' daylight that has a colour temperature of 6,500 K. The CIE Standard Illuminant $D_{65}$ has a correlated colour temperature of $6500 \mathrm{~K}$ and represents average daylight (Fig. 4). Alternative ' $\mathrm{D}$ ' series illuminants are $\mathrm{D}_{50}, \mathrm{D}_{55}$ and $\mathrm{D}_{75}$ corresponding to correlated colour temperatures of $5,000 \mathrm{~K}, 5,500 \mathrm{~K}$

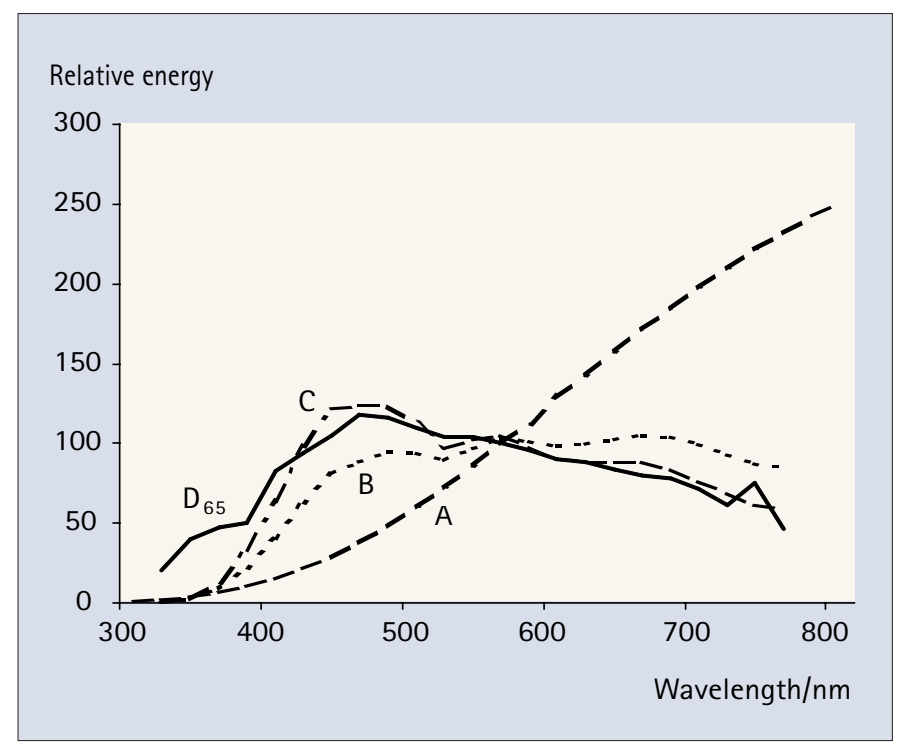

Fig. 4 Spectral energy distributions of Standard Illuminants A, B, C and D65 
and 7,500 K, respectively. In introducing the 'D' series of illuminants, the CIE in effect made redundant the earlier Standard Illuminants B and C.

Artificial daylight is important from both industrial and scientific viewpoints. In terms of industry/business, the most commonly used source of daylight is fluorescent light whereas scientifically/ technically, xenon lamps are important. Both types of lamp differ to the incandescent lamps thus far discussed. While incandescent bodies (eg heated tungsten filaments) emit light in the form of an energy continuum, fluorescent and xenon lamps are examples of gas-discharge tubes which provide line spectra.

\section{Gas discharge tubes}

If a rare gas (neon, xenon or argon) or a metallic vapour (eg sodium or mercury vapour) is discharged with the aid of electricity (ie an electric current is passed through the gas or vapour) the atoms of the gas/vapour become excited and, when they return to their original (ground) state, a portion or all of the excess energy is emitted in the form of light. The energy of this emitted light is characteristic of the gas/vapour and takes the form of a line spectrum that contains some wavelengths. If the pressure in such a gas discharge tube is low then only a few lines are produced; if the pressure inside the tube is increased, the lines broaden and a continuum of radiation is produced.

As an example, high pressure sodium lights, developed in the 1960s, produce characteristically yellow light due to emissions at 589.0 and $589.7 \mathrm{~nm}$. The high pressure mercury light, one of the most commercially important types of discharge tube, has a bluegreen colour due to peaks at 406, 436, 546 and $577 \mathrm{~nm}$. The SED of the high pressure xenon arc lamp is very broad including the ultraviolet, visible and infra red regions of the spectrum. When filtered, the spectrum resembles closely that of standard illuminant $\mathrm{D}_{65}$; consequently, xenon arc light enjoys extensive use in scientific applications.

\section{Fluorescent lights}

Fluorescent tubes, available from the 1930s, are popular, especially with business, because of commercial cost reasons. As Figure 5 shows, a mercury vapour lamp has a bluish tint which comprises several lines in the UV region (approximately 50\% of the total emission is in the UV). To improve the bluish colour of mercury vapour lamp and to increase the amount of light emitted in the visible region, the inside of the tube is coated with fluorescent phosphors which absorb light in the UV region and reemit light in the visible region. Various phosphors are available which produce light that is similar to daylight or incandescence light, giving rise to different fluorescent tubes termed, for example, cool white, warm white deluxe and daylight; such lights have correlated colour temperatures ranging from 3,000 to $6,500 \mathrm{~K}$. The most important artificial daylight lamps are fluorescent lamps.

\section{Prime colour lamps}

In the 1970s it was shown that an acceptable approximation to artificial daylight could be achieved without duplicating the continuous spectrum, using fluorescent lamps which emitted in three regions of the spectrum, namely red, green and blue (ideally $610 \mathrm{~nm}$ for red, $540 \mathrm{~nm}$ for green and $450 \mathrm{~nm}$ for blue).

The resulting prime colour lamps or three-colour lamps, marketed as 'TL84' (Phillips) or 'Ultralume' (Westinghouse) enjoy much usage in retail stores. Although the SED of fluorescent lamps has not yet been standardized by the CIE, temporary, special SEDs referred to as F1 to F12 are used. The twelve lamps belong to three main types of fluorescent lamp, normal, broad band and three band. The use of illuminants F2 (correlated colour temperature of 4,230 K), F7 (correlated colour tempera-

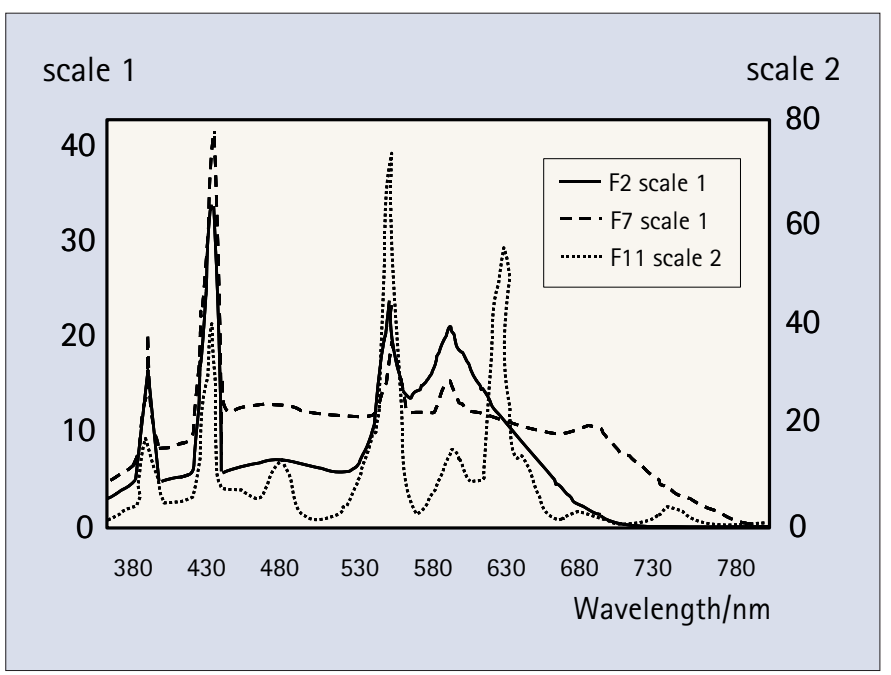

Fig. 5 SED of F type fluorescent lamps

ture of $6,500 \mathrm{~K}$ ) and F11 (correlated colour temperature of $4,000 \mathrm{~K}$ ) is preferred (Fig. 5).

\section{OBJECT}

Light (and all electromagnetic radiation) can interact with objects in various ways. It can be reflected from the surface of a body or it can be absorbed by the body or transmitted through the body (Fig. 6). Light can also be refracted if its speed of propagation changes from one medium (eg air) to another (eg water).

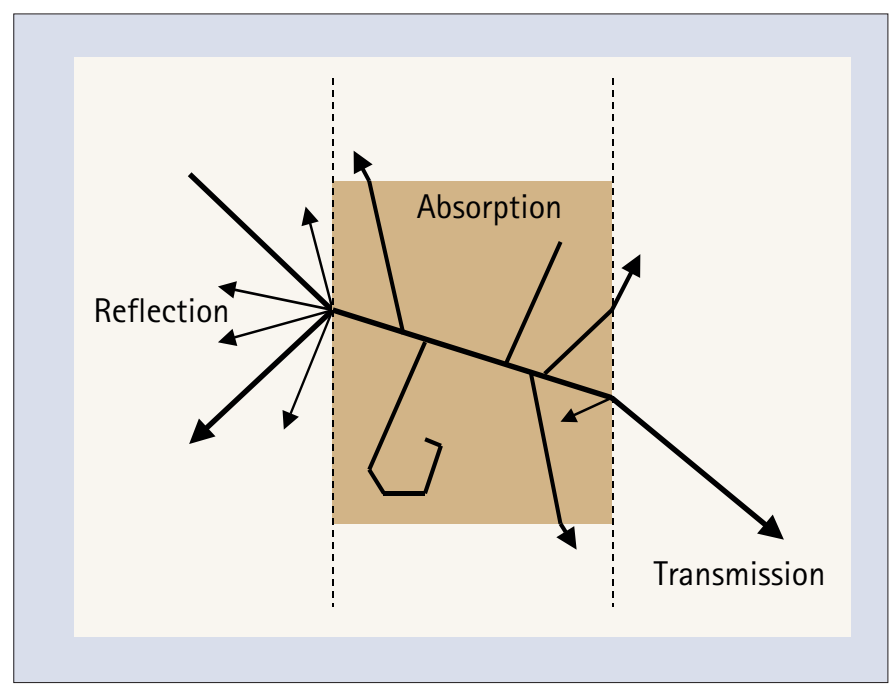

Fig. 6 Simplified representation of reflection, absorption and transmission of light through a translucent material

\section{Reflection}

In the case of any body, if the air to material surface is smooth then we obtain a small amount of specular (mirror-like) reflection at the air/surface boundary. Natural teeth exhibit high specular (gloss) reflection especially when wet, this being manifest as white reflected light. If the surface has roughness then diffuse reflected light is scattered from the boundary (Fig. 7).

\section{Transmission}

Natural teeth are not opaque but, rather, are translucent, which means that any light which enters the body of the tooth is only partly absorbed. The non absorbed light within the body can then be transmitted inside the tooth and undergo scattering before emerging from the tooth. The internally scattered light that emerges from the 


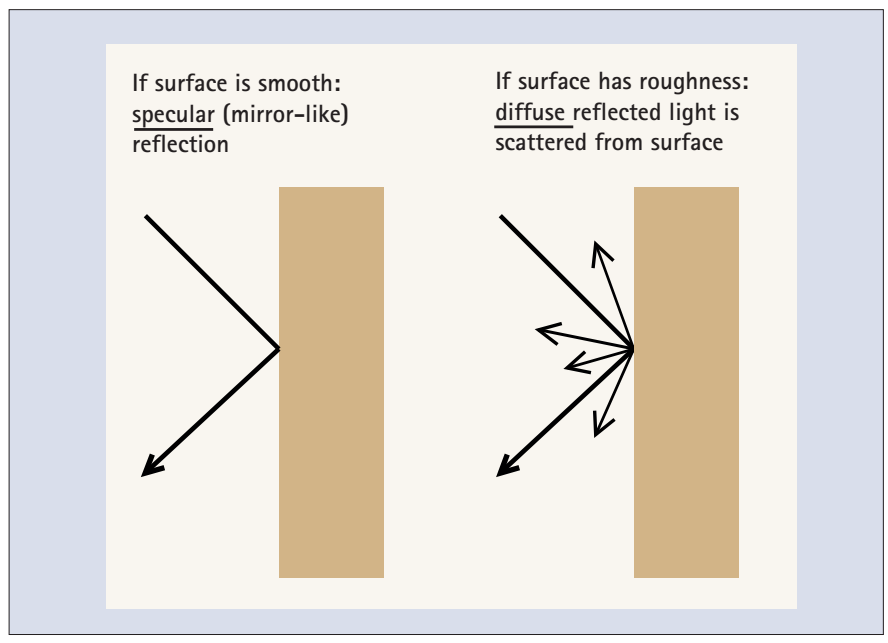

Fig. 7 Specular (a) and specular/diffuse (b) reflection from the surface of an opaque material

tooth contributes to the reflected light; this is termed the body reflectance. This phenomenon is responsible for the characteristic illumination of the gingival region around the tooth.

\section{Absorption}

Any light which is not reflected specularly (or diffusely) enters the opaque body and does not emerge, having been absorbed and converted to heat. Light that has entered the opaque body which is deflected prior to absorption is termed scattered light.

The relative extents of reflection, absorption and transmission of light determine the colour of an object (Fig. 7). The reflection/absorption characteristics of a given material can be measured instrumentally and expressed as the reflectance factor which runs from zero to one (the reflectance factor is the ratio of the amount of light reflected from a sample to that reflected from a reference white standard); in practice, the term is multiplied by 100 and expressed as a percentage. The nomenclature $R(\lambda)$ is commonly used to represent reflectance factor, the $\lambda$ term indicating that the reflectance is wavelength-specific. Also, it is common to use the word reflectance as a collective term rather than reflectance factor. Thus, for example, an ideal black sample absorbs the majority of the incident (white) light falling upon it and reflects very little whereas an ideal white sample reflects the majority of the incident light falling upon it and absorbs very little. In the case of a blue sweater, a plot of reflectance versus wavelength (in the region 400$700 \mathrm{~nm}$; Fig. 8) would produce a curve, typically showing reflection of more blue light (light of shorter wavelength) than yellow or red light which is absorbed. In contrast, the reflectance curve of a

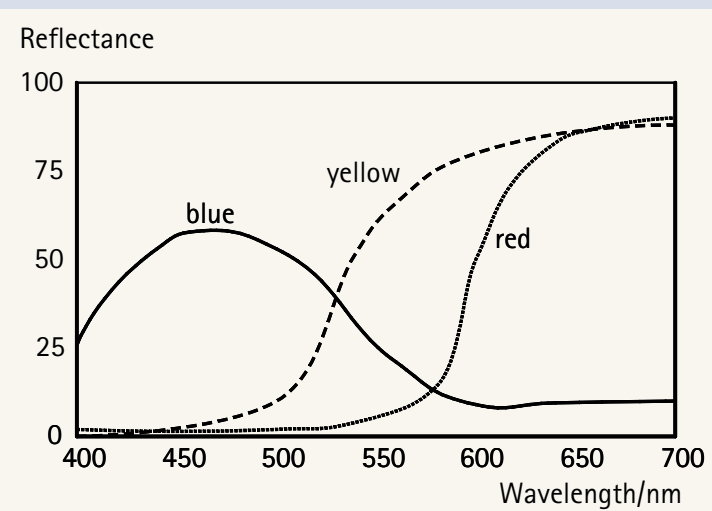

Fig. 8 Reflectance values of typical red, yellow and blue hues typical red hue would show reflectance of more red light than other wavelengths which were absorbed (Fig. 8).

A further characteristic displayed by natural teeth is opalescence. When white light (of a wavelength approx. 400-800 nm; $0.4 \mu \mathrm{m}-$ $0.8 \mu \mathrm{m}$ ) strikes an object of particle size less than the incident wavelength then scattering occurs. In tooth enamel, the composite particles are less than $0.4 \mu \mathrm{m}$ in size and so the blue portion of the incident light is reflected while the red portion of the incident light is transmitted and absorbed giving rise to the bluish tint of illuminated natural teeth.

\section{OBSERVER}

This is the human eye/brain system which constitutes the human colour vision apparatus. In essence, the retina comprises two types of light-sensitive receptors, namely rods and cones. The rods are responsible for scotopic (low light level, monochromatic) vision while cones are responsible for photopic (medium/high light level, full colour) vision. Three types of cone receptor cells (commonly referred to as S (short wavelength), $M$ (medium wavelength) and L (long wavelength)) are present in the retina and vary in their sensitivity to light in three different wavelength bands, peak sensitivities being in the blue (approx. $440 \mathrm{~nm}$ ), green (approx. $545 \mathrm{~nm}$ ) and yellow-green (approx. $585 \mathrm{~nm}$ ) regions of the spectrum. The signals generated by the cone receptor cells are processed by the brain which converts the signals into sensations that we interpret as red, green and blue colours (and their combinations). Cones (and rods) are not uniformly distributed throughout the retina and the fovea, (a small depression in the retina) contains mainly cones at high density. The fovea is the most sensitive part of the eye and is always at the centre of our focus of view. Such is the small size of the fovea that only small samples (of the order of less than $2 \mathrm{~cm}$ viewed at about $25 \mathrm{~cm}$ distance from the eye) will be focused within the fovea area.

\section{Human colour vision}

A variety of colour vision theories have been proposed since the mid-eighteenth century. In 1802 Young proposed that there were three types of photo receptors in the eye and that these were associated with three principal colours, red, blue and yellow (later that year, Young modified his choice of principle, or primary, colours to red, green and violet). This proposal received support through subsequent colour matching experiments by Grassman (1853) and Maxwell (1855, 1860). Von Helmholtz (1852, 1866) developed Young's theory into what is now termed as the Young-Helmholtz-Maxwell Trichromatic theory of colour vision. This theory is based on the fundamental property of colour vision, namely trichromacy, which is the ability to match any colour using an appropriate mixture of three selected (primary) wavelengths.

In 1878, Hering proposed an Opponent Colour theory according to which, any colour can be described in terms of its redness or greenness and yellowness or blueness. As red and green cannot be perceived simultaneously, they are opponent hues. Hering considered that the eye contains two chromatic channels (one for red versus green and one for yellow versus blue) as well as an achromatic channel to account for white versus black.

However, neither the Young-Helmholtz-Maxwell Trichromatic theory nor the Opponent Colour theories can account for all colour vision phenomena and in 1959, Land proposed the Retinex theory in which all colours can be generated by comparing lightnesses through the three cone receptor channels.

None of these three (major) theories provide an adequate explanation of human vision. Modern thinking has involved the combination of the trichromatic and opponent theories in the form of socalled zone theories of colour vision which propose that trichromacy and opponency can occur within different regions of 
the retina. Clearly, human colour vision has not yet been completely explained.

Not all observers have normal colour vision; there are several types of colour vision impairment which affect some $8 \%$ of the population, mostly males. Tests are available to determine the nature of the colour deviancy. ${ }^{8}$

\section{Metamerism}

Metamerism refers to the situation in which two coloured samples match under one set of conditions but not under another. Metamerism relates to a pair of samples viewed simultaneously. For example, two objects may appear to be the same colour when viewed under particular lighting conditions (eg natural daylight) but exhibit distinct colour differences when illuminated under a light source with a different spectral composition, such as tungsten light.

It has been discussed that the perceived colour of an object is determined by the spectral distribution of the light source and the particular reflectance spectrum of the object. If some of the wavelengths that are crucial to the appearance of an object when viewed under a given (first) light source are not present when the object is viewed under a second (different) light source then these wavelengths will not be present in the light reflected from the sample. In this case, the object when viewed under the second light source will be deficient in these wavelengths and thus will be perceived to be of a different colour to that seen when the object is viewed under the first light source. This is an example of illuminant metamerism whereby two objects may match in colour under one illuminant but not under another.

While illuminant metamerism is the most frequent in occurrence, other forms of metamerism arise because of the differences that can occur in the colour vision of observers (observer metamerism) as well as in the angle of view (geometric metamerism) or in the area of view (field size metamerism).

\section{Colour constancy}

If a coloured object is viewed under two different light sources, the perceived colour of the object can remain approximately the same, even though the level and spectral composition (colour) of the two light sources may vary greatly. This is because the human colour vision system is so good in compensating for such changes in illumination, it can adapt to the different types of illumination. Colour constancy is a property associated with an individual coloured object as opposed to metamerism which refers to a pair of coloured objects. Colour constancy is the phenomenon displayed by the majority of natural objects, namely that an object appears to be the same colour when viewed under different light sources.

Colour constancy is intriguing since the spectral distribution of light entering the eye from an object can vary markedly from one light source to another. However, as mentioned, colour constancy is approximate insofar as some objects appear to change markedly in colour from one light source to another; such objects are considered to lack colour constancy.

\section{DESCRIPTION OF COLOUR}

As mentioned earlier, although we can visually distinguish many different colours, it is impossible to define verbally, each of the 3-5 million unique colours that we can perceive. A quantitative method of describing colours is therefore needed. This is colorimetry, or how we can measure, or specify numerically, the sensation of colour perceived by an observer when viewing an object under a particular illuminant. In turn, this requires a means of measuring instrumentally, the colour of an object.

\section{CIE 1931 system}

In 1931, the CIE introduced a system of instrumental colour measurement for describing colours. It is based on the fundamental property of trichromacy (that any colour can be matched using an appropriate mixture of three chosen wavelengths). This approach stems from the pioneering colour mixing experiments of Grassman, von Helmholtz and, especially, Maxwell. The rationale involved is that a colour sensation can be matched by the mixing of three coloured lights or three primary wavelengths (an inherent property of the chosen primary wavelengths is that any one primary cannot be matched by mixing the other two).

If the three primaries, red, green and blue, are represented by $[R],[G]$ and $[B]$ then when these are used to match a given colour [C], the amount of the primaries needed to imitate the colour will be $R, G$ and B. Thus, C units of colour [C] can be matched by $R$ units of the red primary $[R]$ additively mixed with $G$ units of the green primary $[\mathrm{G}]$ and $\mathrm{B}$ units of the blue primary $[\mathrm{B}]$. This is represented as $C[C] \equiv R[R]+G[G]+B[B]$.

The amount of the three primaries used (ie R, G and B) are known as the tristimulus values of the colour [C]. In the $1931 \mathrm{CIE}$ system, a series of (human) observers were employed, using a $2^{\circ}$ viewing angle (so that light illuminated only the fovea). The observers determined the amount of the three primaries that were needed to match all spectral colours within the visible spectrum (in the region 380 to $760 \mathrm{~nm}$ ). The results obtained for the observers were collated and the concept of a standard observer was introduced. Three curves were obtained which represent the colour vision characteristics of the observers because the amount of the three primary lights needed to match the spectral colours was related to the response character of the cone receptors. These curves are known as the colour matching functions of the observer. The 1931 CIE system uses the following terminology.

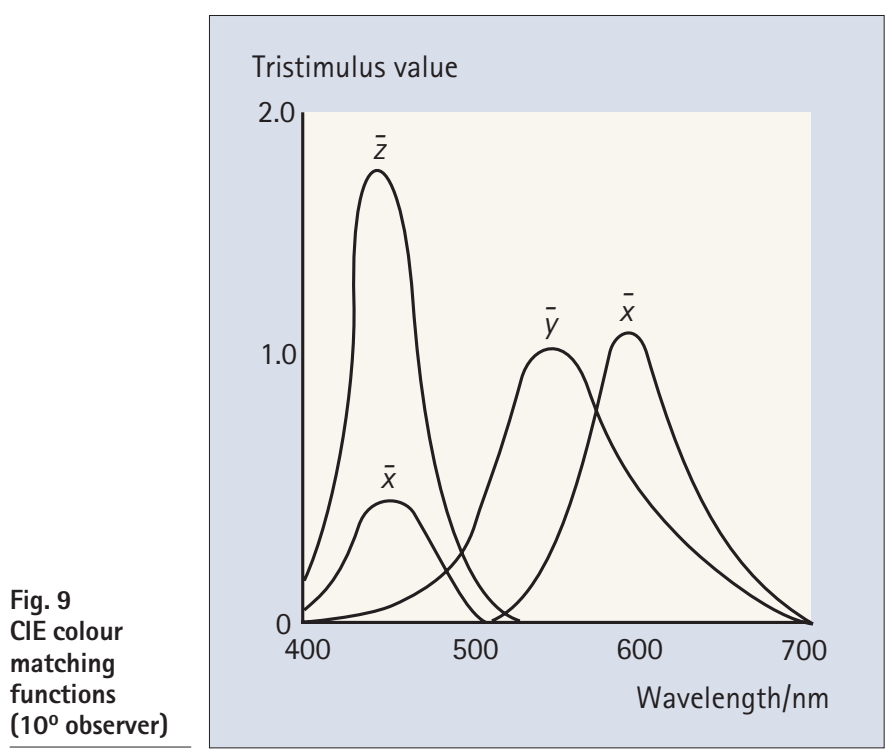

Primaries

Three imaginary primaries are used, specified as [X], [Y] and [Z].

\section{Colour matching functions}

These characterise the response of the cone receptors in the observer and are denoted $\bar{x}, \bar{y}$, and $\bar{z}$ (Fig. 9).

\section{Standard observer}

Although a $2^{\circ}$ viewing angle was initially used, in 1964, the CIE introduced a $10^{\circ}$ standard observer which has been adopted in most countries. 


\section{Tristimulus values}

These are denoted as $\mathrm{X}, \mathrm{Y}$ and $\mathrm{Z}$. It was earlier mentioned that the perception of the colour of an object depends on three elements, namely the light source, the object and the observer. Elaborating this simple concept means that the colour of an object depends on:

- The SED of the light that illuminates the sample $(S)$

- The reflectance characteristics of the sample $(R)$

- The sensitivity of the observer (colour matching functions).

As mentioned, in the CIE system, the tristimulus value is the amount of a primary that will match the colour of a given object. The given colour is matched by:

$$
\mathrm{X}[\mathrm{X}]+\mathrm{Y}[\mathrm{Y}]+\mathrm{Z}[\mathrm{Z}]
$$

As the colour of that object also depends on the characteristics of the illuminant, object and observer, it follows that the tristimulus values are related to $\mathrm{S}, \mathrm{R}$ and $\bar{x}, \bar{y}$, and $\bar{z}$. For a given wavelength, $\lambda$, then:

$$
\begin{aligned}
& X=S_{\lambda} R_{\lambda} \bar{x}_{\lambda} \\
& Y=S_{\lambda} R_{\lambda} \bar{y}_{\lambda} \\
& Z=S_{\lambda} R_{\lambda} \bar{z}_{\lambda}
\end{aligned}
$$

Summing for the whole visible region $\sum$ :

$$
\begin{aligned}
& X=\sum S_{\lambda} R_{\lambda} \bar{x}_{\lambda} \\
& Y=\sum S_{\lambda} R_{\lambda} \bar{y}_{\lambda} \\
& Z=\sum S_{\lambda} R_{\lambda} \bar{z}_{\lambda}
\end{aligned}
$$

380

Thus, if a given coloured sample is illuminated with a known light (a CIE standard illuminant of defined SED (S)) and the reflected light $(R)$ is viewed by a known observer (a CIE standard observer of defined $\bar{x}, \bar{y}$, and $\bar{z}$ ), the tristimulus values can be calculated.

\section{Chromaticity}

As mentioned, colour is three-dimensional, with the three attributes of colour being hue, lightness and chroma. This means that

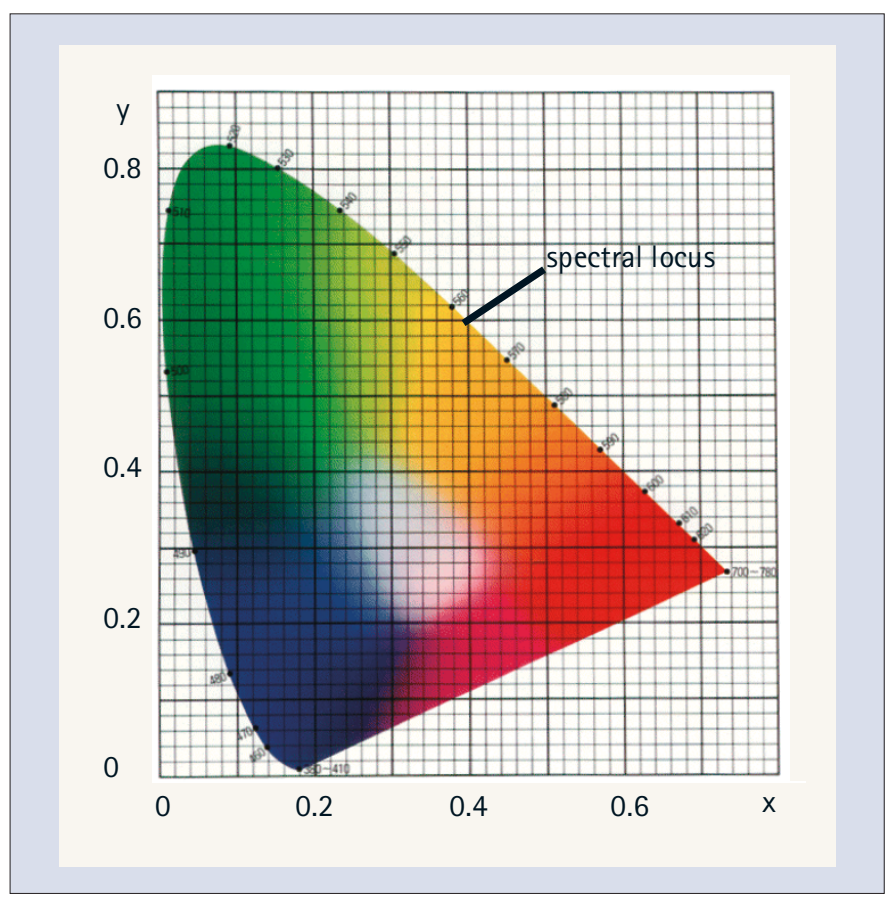

Fig. 10 CIE 1931 chromaticity diagram

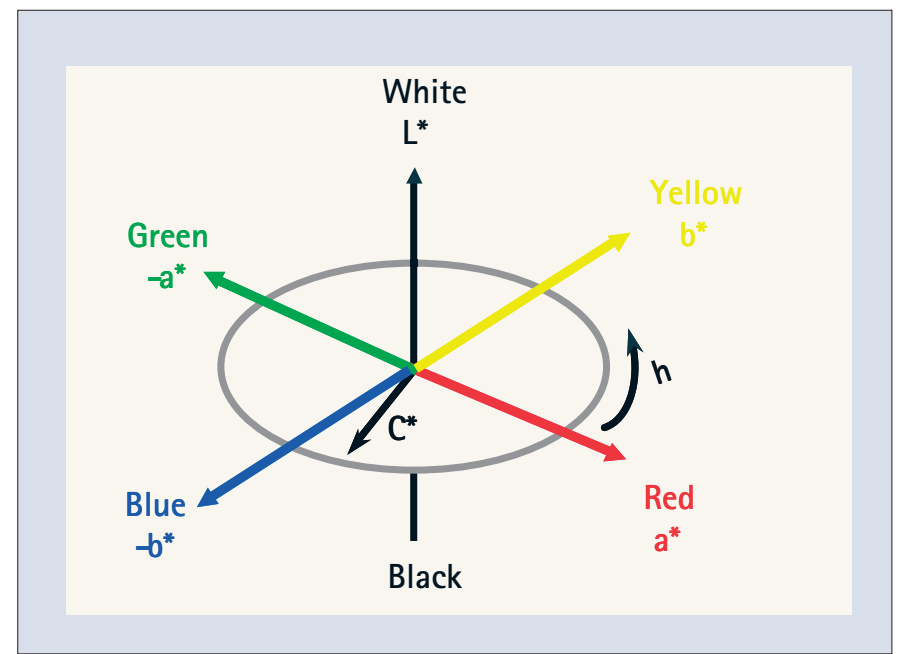

Fig. 11 Representation of $\mathrm{CIEL}^{*} \mathrm{a}^{*} \mathrm{~b}^{*}$ space

the CIE system must be three-dimensional which gives rise to the concept of colour space. In the CIE system, the Y tristimulus value represents the luminance of the object and is normalised to 100 . To represent the other two dimensions of colour, the concept of chromaticity is used.

To represent hue and chroma, chromaticity coordinates are calculated and displayed on a chromaticity diagram (Fig. 10). The

\begin{tabular}{|c|c|c|c|c|}
\hline & \multicolumn{2}{|c|}{$2^{\circ}$ Observer } & \multicolumn{2}{|c|}{$10^{\circ}$ Observer } \\
\hline & A & $\mathrm{D}_{65}$ & A & $\mathrm{D}_{65}$ \\
\hline$x$ & 109.85 & 95.04 & 111.14 & 94.81 \\
\hline$Y$ & 100 & 100 & 100 & 100 \\
\hline Z & 35.59 & 108.88 & 35.2 & 107.3 \\
\hline$x$ & 0.4476 & 0.3127 & 0.4512 & 0.3138 \\
\hline y & 0.4074 & 0.3290 & 0.4059 & 0.3310 \\
\hline
\end{tabular}
chromaticity coordinates are defined as $\mathrm{x}, \mathrm{y}$ and $\mathrm{z}$ where $\mathrm{x}+\mathrm{y}+\mathrm{z}=1$ :

$$
\begin{aligned}
& x=\frac{X}{X+Y+Z} \\
& y=\frac{Y}{X+Y+Z} \\
& z=\frac{Z}{X+Y+Z}
\end{aligned}
$$

In practice a plot of $\mathrm{x}$ versus $\mathrm{y}$ is constructed that exhibits a horseshoe-shaped area and which contains all real colours (Fig. 10).

Neutral colours occupy a central position and moving from this region to the spectral locus the colours increase in chroma. Achromatic colours are represented by a neutral point (Table 1). While the chromaticity diagram represents the hue and chroma of a colour, the third dimension of colour (lightness) is imagined as the Y tristimulus value perpendicular to the plane of the chromaticity diagram. The CIE 1931 colour space contains a horseshoe-shaped region in which all real (perceived) colours can be described numerically.

However, the CIE system is visually non-uniform insofar as equal changes in $\mathrm{x}, \mathrm{y}$ or $\mathrm{Y}$ do not correspond to the same per- 
ceived (visual) difference. This is of great significance with regards to the description of colour difference. Clearly, what is required is for distances in colour space (ie differences between $\mathrm{x}, \mathrm{y}$ or $\mathrm{Y}$ values) to correspond to the same visually perceived differences. Various attempts have been made to improve the 1931 CIE system and in 1976, the CIE proposed the use of the CIE $L^{*} a^{*} b^{*}$ colour space.

\section{CIE L*a*b* colour space}

In this system, which is now far more widely used than the 1931 CIE system, the tristimulus values of the sample are transformed into $\mathrm{L}^{*}$, $\mathrm{a}^{*}$ and $\mathrm{b}^{*}$ coordinates as mutually perpendicular axes (Fig. 11):

$$
\begin{aligned}
& \mathrm{L}^{*}=116\left(\mathrm{Y} / \mathrm{Y}_{\mathrm{n}}\right)^{1 / 3}-16 \\
& \mathrm{a}^{*}=500\left[\left(\mathrm{X} / \mathrm{X}_{\mathrm{n}}\right)^{1 / 3}-\left(\mathrm{Y} / \mathrm{Y}_{\mathrm{n}}\right)^{1 / 3}\right] \\
& \mathrm{b}^{*}=200\left[\left(\mathrm{Y} / \mathrm{Y}_{\mathrm{n}}\right)^{1 / 3}-\left(\mathrm{Z} / \mathrm{Z}_{\mathrm{n}}\right)^{1 / 3}\right]
\end{aligned}
$$

where $X_{n}, Y_{n}$ and $Z_{n}$ are the tristimulus values for a particular standard illuminant and standard observer for a sample reflecting $100 \%$ of the light at all wavelengths. The asterisk simply signifies that CIE defined units have been used as opposed to units from alternative Lab colour space systems.

In this three-dimensional colour space, lightness is represented by $L^{*}$ on a scale of 0 for black to 100 for white. The hue and chroma of a sample are represented on an $a^{*}$ versus $b^{*}$ plot. $a^{*}$ is the red/green coordinate with $+\mathrm{a}^{*}$ indicating red and $-\mathrm{a}^{*}$ indicating green. $b^{*}$ is the yellow/blue coordinate, with $+b^{*}$ indicating yellow and $-b^{*}$ indicating blue. Neutral colours are close to the origin for most illuminants (ie $\left|a^{*}\right|<5,\left|b^{*}\right|<5$ ).

\section{CIEL ${ }^{*} a b^{*}$ colour difference}

The CIE $\mathrm{L}^{*} \mathrm{a}^{*} \mathrm{~b}^{*}$ colour space can be used to specify colour differences via the equation:

$$
\Delta \mathrm{E}_{\mathrm{ab}}^{*}=\left[\left(\Delta \mathrm{L}^{*}\right)^{2}+\left(\Delta \mathrm{a}^{*}\right)^{2}+\left(\Delta \mathrm{b}^{*}\right)^{2}\right]^{0.5}
$$

where $\Delta \mathrm{L}^{*}, \Delta \mathrm{a}^{*}$ and $\Delta \mathrm{b}^{*}$ represent the differences between the corresponding coordinates of two samples. According to this system, a colour difference of one $\Delta \mathrm{E}^{*}{ }_{\text {ab }}$ unit or greater is just perceivable.

\section{CIE $L^{*} C^{*} h^{\circ}$ colour difference}

The cartesian coordinates, $\mathrm{L}^{*}, \mathrm{a}^{*}$ and $\mathrm{b}^{*}$, can be transformed into the cylindrical coordinates $\mathrm{L}^{*}, \mathrm{C}^{*}$ and $\mathrm{h}^{\circ}$ (see later). The lightness $\left(L^{*}\right)$ coordinate is the same as in $L^{*}, a^{*}, b^{*}$ space, with the chroma $\left(\mathrm{C}^{*}\right)$ coordinate being perpendicular to the lightness axis and the hue angle $\left(h^{\circ}\right)$ expressed in degrees $\left(0^{\circ}\right.$ at the $+a^{*}$ axis, $90^{\circ}$ at the $+b^{*}$ axis, $180^{\circ}$ at the $-a^{*}$ axis, $270^{\circ}$ for $-b^{*}$ and back to $360^{\circ}=0^{\circ}$ ) (Fig. 10), where:

$$
\begin{aligned}
& \mathrm{C}^{*}=\left[\left(\mathrm{a}^{*}\right)^{2}+\left(\mathrm{b}^{*}\right)^{2}\right]^{0.5} \\
& \mathrm{~h}=\arctan \left(\mathrm{b}^{*} / \mathrm{a}^{*}\right)
\end{aligned}
$$

Colour difference is expressed in this system by:

$$
\Delta \mathrm{E}_{\mathrm{ab}}^{*}=\left[\left(\Delta \mathrm{L}^{*}\right)^{2}+\left(\Delta \mathrm{C}^{*}\right)^{2}+\left(\Delta \mathrm{H}^{*}\right)^{2}\right]^{0.5}
$$

where $\Delta \mathrm{H}^{*}$ is the metric hue difference as opposed to the angular difference, $\Delta \mathrm{h}^{\circ}$. Colour differences $\left(\Delta \mathrm{E}^{* \mathrm{ab}}\right)$ are the same for any pair of samples whether CIE $\mathrm{L}^{*} \mathrm{a}^{*} \mathrm{~b}^{*}$ or CIE $\mathrm{L}^{*} \mathrm{C}^{*} \mathrm{~h}^{\circ}$ are used.

However, CIE $\mathrm{L}^{*} \mathrm{a}^{*} \mathrm{~b}^{*}$ colour space (and thus CIE $\mathrm{L}^{*} \mathrm{C}^{*} \mathrm{~h}^{\circ}$ colour space) is visually non-uniform with the result that differences between colours at the edges of the colour space may not agree with visually judged colour differences. Consequently, several alternative colour spaces have been devised though none has, as yet, been recommended by the CIE to replace CIE $\mathrm{L}^{*} \mathrm{a}^{*} \mathrm{~b}^{*}$ colour space. In essence, the alternative colour space systems seek to improve the accuracy of colour difference description and modify the $\Delta \mathrm{L}^{*}, \Delta \mathrm{C}^{*}$ and $\Delta \mathrm{H}^{*}$ coordinates obtained using the CIE L*a*b* equations. The best known and most widely used modified colour space systems is the CMC $(l: c)$ formula. ${ }^{9,10}$

\section{CMC colour difference equation}

This formula is based on the same colorimetric principles of the CIE L*a*b* system. The CMC colour difference, $\Delta \mathrm{E}_{\mathrm{CMC}}$ equation is a modification of the CIE $\mathrm{L}^{*} \mathrm{C}^{*} \mathrm{~h}^{\circ}$ colour difference and is preferred as a measure of the acceptability of colour tolerance.

\section{INSTRUMENTAL COLOUR MEASUREMENT}

The functioning of colour measuring instruments mimics that of the human eye in as much as both use red, green and blue (additive) primaries. In this context, additive colour mixing is based on the principle that when the light of the three primary colours, red, green and blue, are superimposed, white light is produced (Fig. 12).

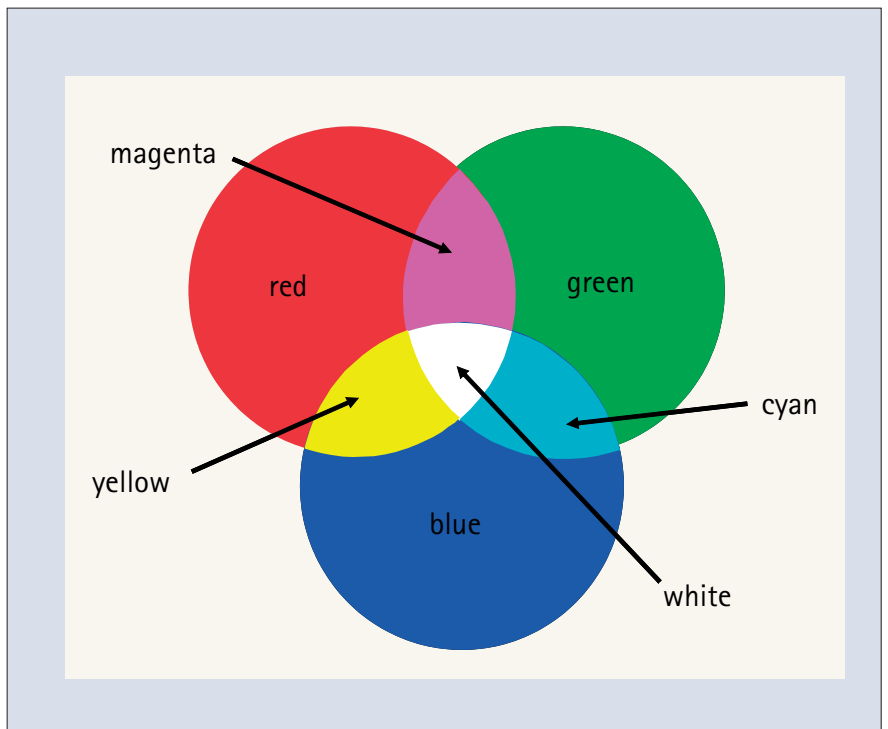

Fig. 12 Additive colour mixing

\section{Colorimeters}

These were the first type of colour measuring instrument and, in essence, attempted to copy the human visual process by using three (or sometimes four) light filters. The earlier colorimeters were visual absorptiometers that were used to visually compare the strength of two coloured transparent solutions. The introduction in the 1930s of the photocell led to the observer's eye being replaced with a light detector. Colorimeters remain in use for the measurement of transparent objects (transmission) and opaque objects (reflectance). In the latter case, the essential features of a colorimeter are a light source (which often is filtered to provide a standard illuminant) that is reflected from the sample and passes through three (or four) filters (which represent the three response curves of the CIE standard observer) and are detected by photodetector. In this way, the colour of a sample can be measured for a particular illuminant/observer combination, such as illuminant $\mathrm{A} / 2^{\circ}$ standard observer.

\section{Spectrophotometers}

These measure the spectral reflectance or transmission of objects and comprise, in essence, a light source, a monochromator and a photodetector. Various light sources are used (eg tungsten halogen bulbs or xenon flash tubes) either filtered or unfiltered, providing light of the required SED. In the case of an opaque sample, the reflectance of the sample is measured, typically in the range 380 to $760 \mathrm{~nm}$, at discrete wavelengths. The 
light is thus resolved into individual wavelengths either before or after it falls on the sample using a monochromator (prism, diffraction grating or interference filter). When measuring an opaque sample using a reflectance spectrophotometer, consideration must be made of both diffuse and specular reflectance and a variety of illuminating and viewing geometries are used.8,9 The detectors used typically are photodiodes.

\section{CONCLUSIONS}

The perception of the colour of an object depends on the combination of the spectral characteristics of the light source, the way in which the object modifies the light reflected from it and, also, the characteristics of the observer. Light sources vary from natural daylight, through fluorescent lights to prime colour lamps. However, although artificial light sources overcome the variability of natural light, as all light sources vary considerably in the amount of radiation emitted at a given wavelength, the colour of different light sources varies markedly. Objects also differ in the ways in which they interact with the light incident upon them and the relative extents of reflection, absorption and transmission of light determine the colour of an object. Whilst the intricacies of human colour vision have not yet been completely unravelled, such is the quality of the eye/brain system that it is capable of fabulous resolution of colour. However, because it is impossible for an observer to define verbally, each of the incredible number of unique colours that can be perceived visually, a quantitative method of describing colours is used: this is colorimetry, which enables the numerical specification of the sensation of colour perceived by an observer when viewing an object under a particular illuminant. In turn, colorimetry uses a system of instrumental colour measurement for describing colours that is grounded in the fundamental property of trichromacy, namely that any colour sensation can be matched by the mixing of three coloured lights. Instrumental colour measurement forms the basis of the three-dimensional, CIE colour space, which is based on the three attributes of colour, hue, lightness and chroma and which embraces the concept of standard observers and standard illuminants. Although several colour spaces have been devised, the CIE $\mathrm{L}^{*} \mathrm{a}^{*} \mathrm{~b}^{*}$ colour space is the most popular and can be used to describe, numerically, the colour difference between two samples, such that a colour difference of one $\Delta \mathrm{E}^{*}{ }_{\mathrm{ab}}$ unit or greater is perceivable. Colour measuring instruments mimic the human eye insofar as they use additive (red, green and blue) primaries. Colorimeters typically use a filtered light source to provide a standard illuminant and additional filters to represent a given standard observer. Although these are still used for the measurement of transmission and reflectance, they have been mostly supplanted by spectrophotometers, which measure the spectral reflectance or transmission of objects and comprise, in essence, a light source, a monochromator and a photodetector.

Concise Oxford Dictionary. 6th ed. Oxford: University Press, 1980.

Nassau K. Color for Science, art and technology. Amsterdam: Elsevier, 1998.

Kuehni R G. Color:An introduction to practice and principles. New York: Wiley, 1997.

. Zollinger H. Color: A multidisciplinary approach.Zurich: Wiley-VCH, 1999.

5. Judd D B, Wyszecki G. Color in business, science and industry. 3rd ed. New York: Wiley, 1975.

6. Burns R S. Billmeyer and Saltzman's principles of color technology. 3rd ed. New York: Wiley, 2000.

7. Nassau K. The physics and chemistry of color: The fifteen causes of color. New York: Wiley, 1983.

8. McDonald R. Colour physics for industry. Bradford: Dyers Co. Pub. Trust, 1987.

9. Berger-Schunn A. Practical color measurement: A primer for the beginner; a reminder for the expert. New York: Wiley, 1994. 